\title{
Políticas de saúde da mulher no ambiente escolar: um exame bibliográfico
}

\author{
Women's health policies in the school environment: a bibliographic examination \\ Políticas de salud de la mujer en el ámbito escolar: un examen bibliográfico
}

Edmar Reis Thiengo ${ }^{1 *}$, Alcilene Batalha dos Santos Mota1 ${ }^{1}$, Jocileia Monteiro', Silas Valério1, Mirielle de Castro Sedano ${ }^{1}$, Patricia de Souza Terra de Martin'1, Josinete Braga Borges Lordes', Silvana Aparecida Faria Santos ${ }^{1}$, Adelma Monteiro Benevides de Lima ${ }^{1}$, Edivan Paes de Freitas ${ }^{1}$.

\section{RESUMO}

Objetivo: É objetivo neste exame bibliográfico, reunir a literatura existente para apresentar as políticas públicas de saúde da mulher no ambiente escolar através dos programas governamentais como o Programa Saúde na Escola (PSE). Revisão Bibliográfica: A saúde da mulher no Brasil assumiu lugar de importância nas casas de saúde pública e particulares, sendo objeto de campanhas e ações que visam reduzir a incidência de mortes femininas. Historicamente a mulher teve sua saúde desprezada, os cuidados reduzidos e o papel na sociedade resumido aos cuidados com o lar, além da objetificação à serviço da figura masculina. Na busca por reduzir os descuidos com a saúde da mulher, campanhas e políticas foram lançadas para aumentar os cuidados e garantir um tratamento mais humanizado às necessidades femininas. Inserir essas políticas de cuidados nas escolas, foi uma forma de garantir que as futuras gerações fossem conscientizadas para as reais necessidades das mulheres, e um meio de alcançar uma grande parcela de mulheres através dos programas públicos de saúde. Considerações finais: O ambiente escolar funciona como instrumento de acesso às mulheres e as escolas ampliam o raio de pacientes, uma forma de prevenir doenças e aumentara conscientização acerca das políticas de saúde.

Palavras-chave: Saúde da Mulher, Saúde na Escola, Saúde.

\begin{abstract}
Objective: It is objective in this bibliographic examination, to gather the existing literature to present public policies for women's health in the school environment through government programs such as the Programa Saúde na Escola (PSE). Bibliographic Review: Women's health in Brazil has assumed an important place in public and private health homes, being the subject of campaigns and actions aimed at reducing the incidence of female deaths. Historically, women have had their health neglected, their care reduced and their role in society reduced to home care, in addition to objectification in the service of the male figure. In the quest to reduce carelessness with women's health, campaigns and policies were launched to increase care and ensure a more humane treatment of women's needs. Inserting these care policies in schools was a way of ensuring that future generations were made aware of the real needs of women, and a means of reaching a large proportion of women through public health programs. Final considerations: The school environment works as an instrument of access to women and schools expand the range of patients, a way to prevent diseases and increase awareness about health policies.
\end{abstract}

Key words: Women's Health, School Health, Health.

\section{RESUMEN}

Objetivo: El objetivo de este examen bibliográfico es reunir la literatura existente para presentar políticas públicas de salud de la mujer en el ámbito escolar a través de programas gubernamentales como el Programa Saúde na Escola (PSE). Revisión bibliográfica: La salud de la mujer en Brasil ha asumido un lugar importante en los hogares de salud públicos y privados, siendo objeto de campañas y acciones dirigidas a

\footnotetext{
${ }^{1}$ Faculdade Vale do Cricaré (FVC), São Mateus - ES. *E-mail: thiengo.thiengo@hotmail.com
} 
reducir la incidencia de muertes femeninas. Históricamente, las mujeres han visto descuidada su salud, reducido su cuidado y reducido su papel en la sociedad al cuidado del hogar, además de la cosificación al servicio de la figura masculina. En la búsqueda por reducir el descuido con la salud de la mujer, se lanzaron campañas y políticas para aumentar la atención y garantizar un tratamiento más humano de las necesidades de la mujer. La inserción de estas políticas de atención en las escuelas es una forma de concienciar a las generaciones futuras de las necesidades reales de las mujeres y un medio de llegar a una gran proporción de mujeres a través de programas de salud pública. Consideraciones finales: El entorno escolar funciona como un instrumento de acceso a las mujeres y las escuelas amplían el abanico de pacientes, una forma de prevenir enfermedades y aumentar la conciencia sobre las políticas de salud.

Palabras clave: Salud de la mujer, Salud escolar, Salud.

\section{INTRODUÇÃO}

Falar da saúde da mulher se tornou comum na contemporaneidade, entretanto nem sempre foi assim. Historicamente a figura feminina gozou de desprezo e ausência de cuidados nas sociedades ocidentais. $O$ machismo histórico construído ao longo dos milênios resultou na necessidade de lutas específicas que sugerem reduzir a desigualdade criada entre homens e mulheres. Ao atentar para as necessidades femininas, surgem as bandeiras relacionadas à saúde da mulher, espaço mais do que justo, já que não se trata de cuidar mais das mulheres do que dos homens, mas sim promover amparo às necessidades que as mulheres possuem e que nem sempre foram prioridades. É dar as mulheres de acordo com suas necessidades, e garantir-Ihes o direito à vida (PRÁ JR e EPPING L, 2012).

Segundo Hillesheim B, et al. (2009), visto que as formas de subjetivação são entendidas como práticas estabelecidas no cotidiano que configuram as formas específicas de as pessoas se relacionarem e se relacionarem, entendemos a área da saúde da mulher como um campo de criação de objetos. Ressaltamos que, no campo da saúde, são criados programas e políticas voltados para a população que adotam configurações específicas de acordo com as articulações estabelecidas em cada momento histórico. Os discursos que configuram as práticas de saúde da mulher hoje são atraídos por verdades que embasam o cuidado com o corpo feminino. O surgimento de discursos/verdades sobre a saúde da mulher no Brasil configura-se por políticas públicas de saúde como o Programa Nacional de Atenção Integral à Saúde da Mulher (PAISM).

A honestidade é um dos princípios orientadores da prática de acordo com a Lei 8080/1990 os serviços que compõem o Sistema Único de Saúde (SUS) para os quais o atendimento deve ser explicitado atividades promocionais, preventivas, de tratamento e de reabilitação, considerando suas dimensões biopsicossocial e combinar continuamente todos os níveis de complexidade no SUS. Nesse sentido, nos debates da II Conferência Nacional de Saúde da Mulher, realizada em 2017, foi apontado que a PNAISM, assim como a Rede Cegonha, tem como objetivo qualificar o pré-natal, parto/nascimento, puerpério e redução da mortalidade materna e infantil (MELOTTI J, et al., 2018). A integralidade, além do princípio constitucional defendido como prerrogativa da humanização da atenção à saúde, busca oportunidades de captação das necessidades humanas mais amplas, valorizando a combinação de atividades preventivas e solidárias.

No entanto, o modelo teórico-conceitual do sistema de saúde, ou seja, a biomedicina, é um importante obstáculo epistemológico à proposta da complexidade. Além disso, existem problemas de saúde associados a contextos sociais, culturais, econômicos e políticos que influenciam o processo de adoecimento e se estendem para além do campo das políticas exclusivamente de saúde, em uma complexidade que impõe constrangimentos e uma multiplicidade de desafios. Soma-se a isso o caráter intersetorial incerto que precisa ser investido, pois as atividades intersetoriais estão além da responsabilidade do setor saúde, indicando a possibilidade de ocorrência de problemas de saúde (COELHO EAC, et al., 2009).

Na promoção da saúde, as ações de educação em saúde podem ser intervenções decisivas, pois são suscitadas pela problemática, análises e proposições dos profissionais de saúde e da comunidade (corpo central do processo). Nessa perspectiva, esta abordagem assume que seu eixo norteador é fortalecer a 
capacidade de escolha do sujeito e que as informações de saúde são formuladas de forma simples e ambiental pois as pessoas podem fazer escolhas de vida mais saudáveis. Portanto, basta eliminar a compreensão da educação em saúde na transferência de informações dos profissionais de saúde aos usuários, pois, como método político de ensino, a educação em saúde requer o desenvolvimento da reflexão. Os temas históricos e sociais sobre a realidade permitem-Ihe autonomia, libertação e decisões saudáveis para o cuidado de si, da família e da comunidade (NUNES JM, et al., 2014).

As políticas públicas de saúde e educacionais compõem os objetivos deste artigo, que é apresenta-las de maneira a instruir socialmente e evidenciar suas importâncias. Além de abrir o debate para possíveis novas políticas de saúde da mulher no ambiente escolar, expondo assim a importância de se trabalhar desde a infância como é importante cuidar do próprio corpo. É também um objetivo claro, apontar as doenças mais frequentes em mulheres e como as ações nas escolas podem agir efetivamente na prevenção das mesmas.

\section{REVISÃO BIBLIOGRÁFICA}

Para Vasconcelos MFF, et al. (2017), enquanto acreditamos na evidência ou na natureza das coisas, em "algo que é causado pelo fato de que sempre existiu" para a saúde da mulher, não há nada natural. As coisas não existem em si mesmas, são apenas correlatos de práticas sociais muito antigas, são produtos sóciohistóricos e culturais. Parece forte lembrar que o que é natural também é construído socialmente e, portanto, que não há natureza das coisas ou pessoas que acontecem sem ser construída por meio de relacionamentos, ciência e interações sociais.

A mulher, então, tem seu corpo, sua subjetividade, sua saúde assumida por práticas e linguagens que falam por ela. Parece que as palavras gênero, saúde, saúde da mulher (mulher) assumiram a forma de prescrição, homogeneização e limitação de formas de vida. Nesse sentido, essas práticas e discursos mostram uma certa forma de pensar e agir sobre aqueles corpos que se relacionam com ações históricas e socialmente construídas, mas que agora são considerados "naturais" e, portanto, generalizáveis, codificando a história única de cada mulher, bem como seus movimentos de singularidade (VASCONCELOS MFF, et al., 2017). No Brasil, na primeira metade da década de 1930, políticas nacionais de saúde foram criadas para ajudar as mulheres a se manterem saudáveis para minimizar a grave situação de saúde a que as mulheres estavam expostas, mas limitadas conforme exigido pela gravidez e parto.

A Organização Mundial da Saúde (OMS), tendo definido o conceito de saúde, leva em consideração os direitos das mulheres à sexualidade e à reprodução humana. Essas propostas foram complementadas pelo desenvolvimento dos princípios e diretrizes do PAISM que acompanham a implantação do SUS. As desigualdades sociais, econômicas e culturais mostram que o processo de adoecer e morrer é particularmente diferente. O número de mulheres que vivem na pobreza é superior ao dos homens; porque as mulheres gastam metade do seu tempo e atividades em casa sem receber nada pelo serviço, limitando o acesso aos bens sociais, incluindo saúde (MATTOS JGS, et al., 2017).

Não é novidade que o vínculo entre saúde e educação é reconhecido. De acordo com o argumento desta estreita ligação, existe pelo menos um consenso entre as duas áreas: um bom nível de educação está associado a uma população mais saudável, e uma população saudável tem mais probabilidade de saber sobre educação formal e informal. Dependendo de onde é falado e de quais diálogos são utilizadas, todos os tipos de discursos e cenários podem ser encontrados, ou, em outras palavras, sob este argumento cabem várias abordagens sobre o assunto. A escola foi um importante ponto de encontro para saúde e educação, proporcionando amplas oportunidades para iniciativas como: atividades diagnósticas clínicas e / ou comunitárias, estratégias de rastreamento e / ou encaminhamento para especialistas em saúde ou atenção primária; atividades no domínio da educação e promoção da saúde (CASEMIRO JP, et al., 2014).

É preciso discutir conceitos que embasam as atividades de saúde como práticas pedagógicas, explicandoos a todos os envolvidos. Os conceitos pedagógicos são diferentes formas de compreensão, teorização e prática da educação, denotando o modo de ação e implementação de um ato educativo. Do ponto de vista 
pedagógico, vários conceitos podem ser divididos em duas tendências principais: a pedagogia tradicional e os conceitos contra hegemônicos. Vale destacar que diversas correntes e práticas pedagógicas estão mais próximas de uma ou de outra tendência. No Brasil, as atividades de educação e saúde voltadas para os alunos estiveram presentes em discursos oficiais de 1889, ou seja, desde a época da Primeira República, voltadas para o ensino de comportamentos e hábitos saudáveis (CARVALHO FFB, 2015).

Para Agathão BT, et al. (2018), os determinantes da saúde e da doença passam pelos campos sociais e psicológicos, especialmente na adolescência, à medida que o indivíduo experimenta e muda. Por envolver a fase de construção da identidade, tais experiências podem levar a comportamentos de risco, configurando seus atributos e atitudes na idade adulta e na velhice. Saber mais sobre como os adolescentes veem suas vidas permite que você entenda melhor sua saúde.

Conforme discutido em alguns estudos internacionais que analisaram a Qualidade de Vida Relacionada à Saúde (QVRS) de alunos adolescentes, este estudo aprofundado pode ser uma ferramenta de gestão, com maneiras de orientar a organização de recursos e a tomada de decisões para melhorar a qualidade de vida dos alunos. Na adolescência, prevalecem os estudos que examinam a percepção da QVRS em pacientes crônicos, desenvolvidos principalmente em regime de internação ou ambulatório. Por outro lado, tem havido um interesse recente em pesquisar grupos considerados saudáveis, portanto realizados em outros contextos (AGATHÃO BT, et al., 2018).

\section{As políticas de saúde da mulher na escola}

A faixa etária mais acometida pelo câncer do colo do útero é de 25 a 60 anos, porém, como o início da vida sexual torna os adolescentes mais vulneráveis aos agravos à saúde reprodutiva e sexual, os adolescentes constituem um grupo altamente vulnerável a essa situação. Nem sempre os adolescentes usam métodos anticoncepcionais durante a primeira relação sexual para protegê-los de uma gravidez indesejada e de DST/AIDS. Estudos têm demonstrado que o HPV (vírus do papiloma humano), o principal carcinógeno do câncer cervical, é transmitido nos estágios iniciais da AIDS. A vida sexual gira em torno da adolescência ou 20 anos. Por isso, as políticas de vacinação nas escolas constituem um importante método de prevenção de doenças em mulheres nas escolas (CIRINO FMSB, et al., 2010).

A adolescência é um período complexo da vida humana, caracterizado por mudanças sociais, psicológicas, anatômicas e hormonais, aliadas a novas experiências, que definem a construção da personalidade dos futuros adultos e contribuem para o desenvolvimento de seus padrões de comportamento e valores pessoais. Por se tratar de um período de fortes contradições psicológicas e sociais, manifesta-se no confronto e oposição aos valores sociais, tradições e posições jurídicas, sendo uma forma de expor sua identidade e autonomia em relação aos adultos, portanto, esses sujeitos podem ser vulneráveis a abusos e violação de comportamento de vida, portanto, é mais vulnerável a danos à saúde. Nesse ponto do ciclo vital, o adolescente geralmente não compreende às regras da sociedade e sua sobrevivência é regida por suas próprias regras, que podem beneficiar o uso de drogas, álcool e sexo inseguro (FIEDLER MW, et al., 2015).

A gravidez na adolescência é considerada um fato precoce nesta fase da vida, com graves consequências como abandono escolar, pondo em risco o feto e a mãe, conflitos familiares, discriminação social, afastamento de grupos sociais, adiamento ou destruição de sonhos e planos. Em decorrência da gravidez na adolescência, podem ocorrer sentimento de perda, tristeza, solidão, isolamento, preocupação além de desemprego ou entrada no mercado de trabalho não qualificado. As ações de prevenção ainda são frágeis, devido ao conservadorismo social que implica uma série de restrições a essas políticas, contudo as informações e instruções, são uma forma de combater a gravidez precoce e todas as possíveis complicações na saúde da mulher, sejam elas físicas ou mentais (FIEDLER MW, et al., 2015).

O estabelecimento de uma relação horizontal entre profissionais de saúde e adolescentes pode proporcionar um novo olhar para a atenção à saúde, incorporando a ideia do adolescente como protagonista na construção dos processos individuais e coletivos de saúde e potencializando a sociedade com potencial de libertação, autonomia e responsabilidade. Dada a dimensão adolescente, é imprescindível atender às 
necessidades contemporâneas de desenvolvimento, bem como ampliar as alternativas de prevenção de situações de vulnerabilidade por meio da educação. É preciso também enfrentar o desafio de utilizar métodos participativos que promovam o papel do adolescente no planejamento e na execução das atividades (COSTA ACPJ, et al., 2015).

Na discussão de temas como consumo de drogas e comportamento sexual, é importante entender que para que a informação tenha um papel preventivo e de promoção da saúde entre os jovens, ela deve ser cuidadosamente articulada para não despertar a curiosidade sobre o consumo ou a compra e despertar uma compreensão para que a vida sexual não comece cedo. Para que a informação seja considerada fator de proteção e promoção da saúde, ela deve ser compartilhada de forma correta e completa para informar sobre os efeitos negativos - uso de drogas e sexo desprotegido - e ainda deve ser mencionado que existe uma breve sensação de prazer, que pode causar sofrimento no futuro. Portanto, acredita-se que os jovens terão condições de agir de forma mais consciente diante das pressões externas (VIERO VSF, et al., 2015).

A educação em saúde visa considerar os princípios do Sistema Único de Saúde (SUS) por meio da promoção da saúde e da conscientização das pessoas e comunidades que buscam a proteção de seus direitos. Portanto, para mudar o comportamento dos indivíduos, a expressão de meios que articulem educação e saúde ajuda a evitar o risco de impedir que as pessoas tenham uma vida saudável (BESERRA EP, et al., 2011).

Neste contexto de vulnerabilidade, os jovens distinguem-se pelo facto de se encontrarem numa fase delicada da sua orientação comportamental, exigindo muitos temas, como a sexualidade, para serem abordados, visto que na maioria dos casos os jovens são imaturos e alguns deles são aventureiros, ignorando a possibilidade de contrair alguma doença sexualmente transmissível (DST), e até acreditar que mantêm relações sexuais com pessoas que estão seguras, livres de doenças infecciosas, quando na verdade todos são suscetíveis à infecção (BESERRA EP, et al., 2011).

Os efeitos das doenças sexualmente transmissíveis possuem uma grande incidência sobre as mulheres, já que o comprometimento da saúde mental também deve ser levado em consideração, e gravidez precoce, contrair DSTs e tomar para si, bem cedo, certas responsabilidades, gera um comprometimento tremendo diante de inúmeras situações. A sociedade tem uma cobrança maior para mulheres, a pressão que se instalou mediante o machismo estrutural é um outro grande problema que precisa ser trabalhado em todos os campos da sociedade, pois de uma certa forma gera danos à saúde das mulheres (RAMOS LS, et al., 2020).

\section{Principais doenças que atingem as adolescentes e a importância das ações de saúde na escola}

A noção de que os adolescentes raramente adoecem e o hábito de alegar qualquer problema de saúde, físico ou mental, como "normal" ou característico desta fase da vida, limita a atenção à saúde do adolescente e, portanto, avança nas pesquisas nesta área. Porém, durante a adolescência, que dura cerca de dez anos, a pessoa fica mais exposta a determinadas condições e eventos com graves consequências para a saúde, como gravidez precoce, início do uso de drogas lícitas e ilícitas, violência em suas diversas formas, doenças sexualmente transmissíveis, entre outras. O combate às doenças infecciosas por meio da vacinação, do uso de antibióticos e da melhoria do saneamento básico tem levado a mudanças significativas no perfil da doença nas últimas décadas. $O$ contexto ambiental e socioeconômico, os hábitos e estilo de vida são determinantes do estado de saúde de uma população (BRAZ M, et al., 2013).

A adolescência é um período em que a saúde pode ser promovida, pois, além da aquisição de novos hábitos de vida com consequências de curto e longo prazo, diversas mudanças físicas, mentais e sociais irão determinar os padrões de saúde futuros. As evidências sugerem que a intervenção precoce e apropriada durante a adolescência oferece benefícios socioeconômicos e de saúde em longo prazo. A Organização Mundial da Saúde (OMS) está cada vez mais reforçando a importância de serviços de saúde "amigos dos adolescentes". Trabalhar principalmente na adolescência é fundamental para a saúde pública na melhoria do panorama global da saúde, especialmente em áreas como saúde mental, acidentes e doenças crônicas, que vêm ganhando importância (ABREU N, et al., 2018). 
O papilomavírus humano (HPV) é reconhecido como causa de câncer cervical e está associado a vários outros tipos de câncer. Uma revisão recente da literatura estimou a incidência de HPV em 32,1\% entre 576.281 mulheres, variando de $42,2 \%$ em países em desenvolvimento até $22,6 \%$ nos países desenvolvidos. Estima-se que 9 a 10 milhões de pessoas tenham o vírus e que 700.000 novos casos ocorram a cada ano.

Uma revisão da literatura com estudos realizados em mulheres de diferentes regiões do país mostrou a prevalência do HPV de $14,0 \%$ a $54 \% 0 \%$ nas mulheres em geral e de $10,0 \%$ a $24,0 \%$ nas mulheres assintomáticas (OSIS MJD, et al., 2014). De acordo com pesquisa realizada por Carvalho AMC, et al. (2019), a OMS recomenda o uso da vacina contra o papilomavírus humano (HPV), principalmente na população feminina de 9 a 14 anos, antes da atividade sexual.

A evidência mostra que a imunização de meninas (> 80\%) reduz o risco de infecção por HPV em meninos. As vacinas são imunogênicas e eficazes na prevenção do câncer do colo do útero, que é causado principalmente pelos HPV 16 e 18. Ainda para Carvalho AMC, et al. (2019), o esquema de vacinação recomendado é de duas doses com intervalo de seis meses entre a primeira e a segunda dose em pessoas de 9 a 14 anos. Uma pausa de não mais de 12-15 meses é sugerida para completar o esquema de vacinação antes da atividade sexual. Se o intervalo entre as doses for inferior a cinco meses, a terceira dose deve ser administrada pelo menos seis meses após a primeira dose. O esquema de imunização de três doses $(0,1-2$, 6 meses) é recomendado para pessoas com mais de 15 anos de idade e aqueles que estão imunocomprometidos ou infectados com o vírus da imunodeficiência (HIV).

De acordo com a OMS, uma em cada cinco pessoas no mundo é um adolescente de 10 a 19 anos. A população mundial de adolescentes já ultrapassou um bilhão e anualmente 60 em cada 1000 meninas nessa faixa etária tornam-se mães, o que corresponde ao nascimento de 17 milhões de crianças por ano. A adolescência é um período de grandes mudanças físicas e mentais que caracterizam, principalmente nas culturas ocidentais, a transição da infância para a idade adulta. A maturação sexual é acompanhada por reações emocionais mistas (ansiedade, medo, excitação, prazer) e frequentes mudanças de humor, alternando com desânimo e entusiasmo. Os níveis de estresse em adolescentes também aumentam à medida que a sociedade se torna mais complexa e requer mecanismos psicológicos adaptativos mais extensos (CAPUTO VG e BORDIN IA, 2007).

A adolescência é uma fase caracterizada pela transição da infância para a idade adulta, período marcado por grandes mudanças físicas, cognitivas e sociais. A gravidez durante este período é um fator adicional com o qual as adolescentes precisam lidar. Os pesquisadores apontam que as vivências emocionais das adolescentes relacionadas à gravidez e à maternidade foram apresentadas de forma heterogênea, apresentando diferentes padrões de percepção: às vezes positivas com manifestações de satisfação, ganhos emocionais e afirmação da autoestima; às vezes negativo, com tendência a ficar deprimido e perceber a maternidade como uma experiência difícil e solitária (ROSSETTO MS, et al., 2014).

Muitas questões de doenças que atingem potencialmente mulheres são tratadas nas escolas, mas muitas outras ainda precisam de uma maior ênfase e mais cuidados, não por se tratar de problemas com maior acometimento na adolescência, mas para despertar maiores atenções desde cedo, e não permitir que se torne um problema no futuro por falta de conhecimento. Um desses problemas é o câncer de mama, pois muitas mulheres não percebem a importância de se fazer o exame e diagnosticar precocemente a doença, $\mathrm{e}$ muitas não o fazem por falta de conhecimento (RAMOS BF e LUSTOSA MA, 2009).

\section{CONSIDERAÇÕES FINAIS}

Ao concluir as análises literárias acerca da importância de se trabalhar saúde da mulher nas escolas e quais políticas já são executadas, conclui-se que muito ainda há que se trabalhar, mas é importante dar continuidade e ampliar as políticas já existentes, não apenas na intenção de diagnóstico no presente, mas para instigar cuidados indispensáveis na vida adulta. A adolescência é uma fase complexa que precisa de amparo profissionais e intervenções mais assertivas, com a finalidade de garantir o conhecimento sobre assuntos que ainda são tabus para a sociedade. $O$ ambiente escolar se torna, portanto, um espaço propício para se trabalhar essas políticas, já que é o principal canal de acesso do Estado às gerações futuras. 


\section{REFERÊNCIAS}

1. ABREU N, et al. Quais os diagnósticos mais frequentes na adolescência? A realidade de uma consulta de Medicina do Adolescente. Einstein, 2018; 16(2): 1-7.

2. AGATHÃO BT, et al. Qualidade de vida relacionada à saúde de adolescentes escolares. Ciência \& Saúde Coletiva, 2018; 23(2): 659-668.

3. BESERRA EP, et al. Pedagogia freireana como método de prevenção de doenças. Ciência \& Saúde Coletiva, 2011; 16(1): 1563-1570.

4. BRAZ M, et al. Saúde dos adolescentes: um estudo de base populacional em Campinas, São Paulo, Brasil. Cadernos de Saúde Pública, 2013; 29(9): 1877-1888.

5. CAPUTO VG, BORDIN IA. Problemas de saúde mental entre jovens grávidas e não-grávidas. Revista de Saúde Pública, 2007; 41(4): 573-581.

6. CARVALHO AMC, et al. Adesão à vacina HPV entre os adolescentes: revisão integrativa. Texto \& Contexto Enfermagem, 2019; 28(1): 1-8.

7. CARVALHO FFB. A saúde vai à escola: a promoção da saúde em práticas pedagógicas. PhysisRevista de Saúde Coletiva, 2015; 25(4): 1207-1227.

8. CIRINO FMSB, et al. Conhecimento, atitude e práticas na prevenção do câncer de colo uterino e hpv em adolescentes. Escola Anna Nery, 2010; 14(1): 126-134.

9. CASEMIRO JP, et al. Promover saúde na escola: reflexões a partir de uma revisãosobre saúde escolar na América Latina. Ciência \& Saúde Coletiva, 2014; 19(3): 829-840.

10. COELHO EAC, et al. Integralidade do cuidado à saúde da mulher: limites da prática profissional. Escola Anna Nery, 2009; 13(1): 154-160.

11. COSTA ACPJ, et al. Protagonismo de adolescentes na prevenção de doenças sexualmente transmissíveis. Acta Paulista de Enfermagem, 2015; 28(5): 482-487.

12. FIEDLER MW, et al. A prevenção da gravidez na adolescência na visão de adolescentes. Texto \& Contexto Enfermagem, 2015; 24(1): 30-37.

13. HILLESHEIM B, et al. Saúde da mulher e práticas de governo no campo das políticas públicas. Psicologia em Revista, 2009; 15(1): 196-211.

14. MATTOS JGS, et al. A enfermagem no âmbito da saúde da mulher: uma revisão integrativa, Revista Acta Científica, 2017; 1(8): 1-15.

15. MELOTTI J, et al. A PNAISM e a rede cegonha como políticas de saúde: atenção integral à saúde da mulher?. Seminário de políticas públicas e sociais, 2018; 1(1): 39-40.

16. NUNES JM, et al. Prática educativa com mulheres da comunidade: prevenção da gravidez na adolescência. Texto Contexto Enferm, 2014; 23(3): 791-798.

17. OSIS MJD, et al. Conhecimento e atitude de usuários do SUS sobre o HPV e as vacinas disponíveis no Brasil. Prática de Saúde Pública, 2014; 48(1): 123-133.

18. PRÁ JR, EPPING L. Cidadania e feminismo no reconhecimento dos direitos humanos das mulheres. Revista Estudos Feministas, 2012; 20(1): 33-51.

19. RAMOS BF, LUSTOSA MA. Câncer de mama feminino e psicologia. Revista da SBPH, 2020; 12(1): 85-97.

20. RAMOS LS, et al. A saúde na escola como meio de prevenção da gravidez na adolescência: uma breve análise. Revista Eletrônica Acervo Saúde, 2020; 45(1): 1-7.

21. ROSSETTO MS, et al. Maternidade na adolescência: indicadores emocionais negativos e fatores associados em mães de 14 a 16 anos em Porto Alegre, RS, Brasil. Ciência \& Saúde Coletiva, 2014; 19(10): 4235-4246.

22. VASCONCELOS MFF, et al. Saúde da mulher: o que é poderia ser diferente?. Revista Psicologia Política, 2017; 17(39): 327-339.

23. VIERO VSF, et al. Educação em saúde com adolescentes: análise da aquisição de conhecimentos sobre temas de saúde. Escola Anna Nery, 2015; 19(3): 484-490. 